Article

\title{
A New Proof of the Existence of Nonzero Weak Solutions of Impulsive Fractional Boundary Value Problems
}

\author{
Asma Alharbi ${ }^{1,+}$, Rafik Guefaifia ${ }^{2,+}$ and Salah Boulaaras ${ }^{1,3, *,+}$ (C) \\ 1 Department of Mathematics, College of Sciences and Arts, ArRass, Qassim University, \\ Buraidah 51452, Saudi Arabia; ao.alharbi@qu.edu.sa \\ 2 Department of Mathematics, Faculty of Exact Sciences, University Tebessa 12002, Tebessa 12002, Algeria; \\ rafik.guefaifia@univ-tebessa.dz \\ 3 Laboratory of Fundamental and Applied Mathematics of Oran (LMFAO), University of Oran 1, \\ Ahmed Benbella, Oran 31000, Algeria \\ * Correspondence: saleh_boulaares@yahoo.fr or S.Boularas@qu.edu.sa; Tel.: +966-559-618-327 \\ + These authors contributed equally to this work.
}

Received: 15 April 2020; Accepted: 20 May 2020; Published: 25 May 2020

check for updates

\begin{abstract}
The paper deals with the existence of at least two non zero weak solutions to a new class of impulsive fractional boundary value problems via Brezis and Nirenberg's Linking Theorem. Finally, an example is presented to illustrate our results.
\end{abstract}

Keywords: weak solutions; impulsive fractional boundary value problems; Brezis and Nirenberg's Linking Theorem

\section{Introduction}

The paper deals with the existence of weak solutions to the following boundary value problems for impulsive fractional Hamiltonian differential equations:

$$
\left\{\begin{array}{l}
{ }_{t} D_{T}^{\alpha}\left({ }_{0}^{c} D_{t}^{\alpha} u(t)\right)=-\nabla F(t, u), t \in[0, T], \\
\triangle\left({ }_{t} D_{T}^{\alpha-1}\left({ }_{0}^{c} D_{t}^{\alpha} u^{i}\right)\left(t_{j}\right)\right)=\frac{1}{2} I_{i j}\left(u^{i}\left(t_{j}\right)\right), \quad i \in A, j \in B \\
u(0)=u(T)=0,
\end{array}\right.
$$

where $0 \leq \alpha<1,{ }_{0}^{c} D_{t}^{\alpha}$ and ${ }_{t} D_{T}^{\alpha}$ denote the left Caputo fractional derivative and the right Riemann-Liouville fractional derivative of order $\alpha$, respectively,

$$
\begin{aligned}
& T>0,, A:=\{1, \ldots, N\}, B:=\{1, \ldots, L\}, \\
& u(t)=\left(u^{1}(t), u^{2}(t), \ldots, u^{N}(t)\right), \\
& 0=t_{0}<t_{1}<t_{2}<\cdots<t_{L}<t_{L+1}=T,
\end{aligned}
$$

$\nabla F(t, x)$ denotes the gradient of $F(t, x)$ in $x$, and $\triangle$ the operator is defined as

$$
\triangle\left({ }_{t} D_{T}^{\alpha-1}\left({ }_{0}^{c} D_{t}^{\alpha} u^{i}\right)\left(t_{j}\right)\right)={ }_{t} D_{T}^{\alpha-1}\left({ }_{0}^{c} D_{t}^{\alpha} u^{i}\right)\left(t_{j}^{+}\right)-{ }_{t} D_{T}^{\alpha-1}\left({ }_{0}^{c} D_{t}^{\alpha} u^{i}\right)\left(t_{j}^{-}\right),
$$


where

$$
\begin{array}{ll}
{ }_{t} D_{T}^{\alpha-1}\left({ }_{0}^{c} D_{t}^{\alpha} u^{i}\right)\left(t_{j}^{+}\right)= & \lim _{t \rightarrow t_{j}^{+}}{ }_{t} D_{T}^{\alpha-1}\left({ }_{0}^{c} D_{t}^{\alpha} u^{i}\right)(t) \\
& \text { and } \\
{ }_{t} D_{T}^{\alpha-1}\left({ }_{0}^{c} D_{t}^{\alpha} u^{i}\right)\left(t_{j}^{-}\right)= & \lim _{t \rightarrow t_{j}^{-}}{ }_{t} D_{T}^{\alpha-1}\left({ }_{0}^{c} D_{t}^{\alpha} u^{i}\right)(t)
\end{array}
$$

and ${ }_{0}^{c} D_{t}^{\alpha}$ is the left Caputo fractional derivatives of order $\alpha . I_{i j}: \mathbb{R} \rightarrow \mathbb{R}, i \in A, j \in B$ are continuous with $F:[0, T] \times \mathbb{R}^{N} \rightarrow \mathbb{R}$ satisfies the following condition:

(A) $F(t, u)$ is measurable according to $t$ for any $u \in \mathbb{R}^{N}$ and continuously differentiable according $u$ for a.e. $t \in[0, T]$. However, there exist $a \in C\left(\mathbb{R}^{+}, \mathbb{R}^{+}\right)$and $b \in L^{1}\left([0, T], \mathbb{R}^{+}\right)$, such that

$$
|F(t, u)| \leq a(|u|) b(t),|\nabla F(t, u)| \leq a(|u|) b(t)
$$

for any $u \in \mathbb{R}^{N}$ and a.e. $t \in[0, T]$.

Fractional differential equations have recently proved to be valuable tools for modeling many phenomena in various fields of science and engineering. Indeed, one can find many applications in viscoelasticity, electrochemistry, control, porous media, electromagnetic, etc., for example, see [1-19]. Moreover, recently, the existence of solutions of boundary value problems for Fractional differential equations have widely been studied in many papers and we refer the reader to the papers [3-18] and the references therein. For instance, in [3], the authors created a variable structure and, using the critical point theory, investigated the existence of multiple solutions for a class of fractional advection-dispersion equations derived from symmetric mass flow transmission. In [20], Torres consider the fractional Hamiltonian system given by

$$
\left\{\begin{array}{l}
-{ }_{t} D_{T}^{\alpha}\left({ }_{0} D_{t}^{\alpha} u(t)\right)=\nabla F(t, u(t)), \quad t \in[0, T], \\
u(0)=u(T)=0,
\end{array}\right.
$$

where $\alpha \in\left(\frac{1}{2}, 1\right), u \in \mathbb{R}^{N}$, and $F:[0, T] \times \mathbb{R}^{N} \rightarrow \mathbb{R}$ satisfies some conditions. In addition by using a modified version of mountain pass theorem for functional bounded from below due to Bonanno [21], the author studied the existence of at least three different solutions for problem (2) (see Theorems 1 and 2). In addition, under certain conditions and using some critical point theorems In [22] Zhou et al. proved the following fractional Hamiltonian system with impulsive effects has at least one weak solution:

$$
\left\{\begin{array}{l}
{ }_{t} D_{T}^{\alpha}\left({ }_{0}^{c} D_{t}^{\alpha} u(t)\right)=\nabla F(t, u(t)), \quad t \in[0, T], \\
u(0)=u(T)=0, \\
\triangle\left({ }_{t} D_{T}^{\alpha-1}\left({ }_{0}^{c} D_{t}^{\alpha} u^{i}\right)\left(t_{j}\right)\right)=I_{i j}\left(u^{i}\left(t_{j}\right)\right), i=1, \ldots, N, j=1, \ldots, p .
\end{array}\right.
$$

The outline of the paper is as follows: In Section 2, we lay down preliminaries and assumptions, some of which will be needed in the body of the paper. Then, in Section 3, the main result is obtained, which gives the result of the existence of at least two non zero weak solutions to problem (1) via Brezis and Nirenberg's Linking theorem (see Lemma 2 in [23]).

\section{Preliminaries and Assumptions}

In this section, we present some assumptions, definitions and basic properties of fractional computing, used later in this article. For omitted evidence, we refer the reader to ([24]) or other texts on the basic fractional calculation. 
To state our main results, we set

$$
\omega:=\frac{2 \pi}{T}, \xi:=\frac{T^{\alpha-\frac{1}{2}}}{\Gamma(\alpha) \sqrt{2 \alpha-1}} \text { and } t \in[0, T],
$$

where $\Gamma$ is the standard gamma function given by

$$
\Gamma(z)=\int_{0}^{+\infty} z^{\alpha-1} e^{-z} d z .
$$

Now, we assume that $I_{i j}(s)$ and $F(t, u)$ satisfy the following assumptions:

(h1) There exists a constant $\gamma>0$, such that

$$
\lim _{|u| \rightarrow \infty} \inf \frac{F(t, u)}{|u|^{2}} \geq \gamma \text { uniformly for a.e.t } \in[0, T] .
$$

(h2) There exist constants $\rho>0, \lambda>0$ and $k \geq 1$ verify

$$
F(t, u) \leq-\frac{1}{2} \lambda k^{2} \omega^{2}\left(\frac{1}{\Gamma(2-\alpha)}\right)^{2}|u|^{2},
$$

for all $|u| \leq \rho$ and a.e. $t \in[0, T]$.

(h3) There exist constants $\gamma_{i j} \in[0,1)$ for any $i \in A, j \in B$, satisfying

$$
\gamma_{i j} \int_{0}^{\tau} I_{i j}(s) d s \leq I_{i j}(\tau) \tau \text {, for every } \tau \in \mathbb{R} .
$$

(h4) $I_{i j}$ satisfy $c_{i j}:=\inf _{|\tau|=1} \int_{0}^{\tau} I_{i j}(s) d s>0$.

(h5) There exist constants $\mu_{i j}>0$ with an integer $k \geq 1$, which is defined in (h2) for all $i \in A$, $j \in B$, satisfying

$$
0<\int_{0}^{z} I_{i j}(s) d s \leq \mu_{i j} k^{2} \omega^{2}|z|^{2}, \text { for every } z \in \mathbb{R} .
$$

Theorem 1. Suppose condition $(A)$ is satisfied. Suppose that one of the following two conditions is true:

$(H 1)(h 1),(h 3)$ and $(h 4)$ hold with $\gamma>\frac{1}{2}$;

(H2) (h1), (h3) and (h4) hold with

$$
\xi^{2} \sum_{i=1}^{N} \sum_{j=1}^{L} \frac{c_{i j}}{2}<\gamma \leq \frac{1}{2}
$$

In addition, we assume also that the following condition holds:

(H3) (h2), (h3) and (h5) hold with

$$
\lambda \geq 4 \frac{T^{2-2 \alpha}}{3-2 \alpha}+L N \mu \xi^{2}+4 L k^{2} \omega^{2} \mu \xi^{2} \frac{T^{2-2 \alpha}}{3-2 \alpha}
$$

where $\mu=\max _{i \in A, j \in B}\left\{\mu_{i j}\right\}$. Then, problem (1) has at least two nonzero weak solutions. 
Definition 1. ([24]) Let $u$ be a function defined on interval $[a, b]$. left and right Riemann-Liouville fractional integrals of order $\alpha>0$ for a function $u$ denoted by ${ }_{a} D_{t}^{-\alpha} u(t)$ and ${ }_{t} D_{b}^{-\alpha} u(t)$, respectively, are defined by

$$
{ }_{0} D_{t}^{-\alpha} u(t)=\frac{1}{\Gamma(\alpha)} \int_{0}^{t}(t-s)^{\alpha-1} u(s) d s, \quad t \in[a, b]
$$

and

$$
{ }_{t} D_{b}^{-\alpha} u(t)=\frac{1}{\Gamma(\alpha)} \int_{t}^{b}(s-t)^{\alpha-1} u(s) d s, t \in[a, b]
$$

Definition 2. ([24]) Let $u$ be a function defined on $[a, b]$. The left and right Riemann-Liouville fractional derivatives of order $\alpha$ for a function $u$ denoted $b y_{a} D_{t}^{\alpha} u(t)$ and ${ }_{t} D_{b}^{\alpha} u(t)$ are defined by

$$
{ }_{a} D_{t}^{\alpha} u(t)=\frac{d^{n}}{d t^{n}}{ }_{a} D_{t}^{\alpha-n} u(t)=\frac{1}{\Gamma(n-\alpha)} \frac{d^{n}}{d t^{n}}\left(\int_{a}^{t}(t-s)^{n-\alpha-1} u(s) d s\right), t \in[a, b]
$$

and

$$
{ }_{t} D_{b}^{\alpha} u(t)=(-1)^{n} \frac{d^{n}}{d t^{n}}{ }_{t} D_{b}^{\alpha-n} u(t)=\frac{1}{\Gamma(n-\alpha)} \frac{d^{n}}{d t^{n}}\left(\int_{t}^{b}(s-t)^{n-\alpha-1} u(s) d s\right), t \in[a, b]
$$

where $n-1 \leq \alpha<1$ and $n \in \mathbb{N}$. In particular, if $0 \leq \alpha<1$, then

$$
{ }_{a} D_{t}^{\alpha} u(t)=\frac{d}{d t}{ }_{a} D_{t}^{\alpha-1} u(t)=\frac{1}{\Gamma(1-\alpha)} \frac{d}{d t}\left(\int_{a}^{t}(t-s)^{-\alpha} u(s) d s\right), t \in[a, b]
$$

and

$$
{ }_{t} D_{b}^{\alpha} u(t)=-\frac{d}{d t}{ }_{t} D_{b}^{\alpha-1} u(t)=-\frac{1}{\Gamma(1-\alpha)} \frac{d}{d t}\left(\int_{t}^{b}(s-t)^{-\alpha} u(s) d s\right), t \in[a, b]
$$

Definition 3. ([24]) Let $\alpha \geq 0$ and $n \in \mathbb{N}$, we have:

(i) If $\alpha \in\left[n-1,1\right.$ (and $u \in A C^{n}\left([a, b], \mathbb{R}^{n}\right)$, then the left and right Caputo fractional derivatives of order $\alpha$ for function $u$ denoted by ${ }_{a}^{c} D_{t}^{\alpha} u$ and ${ }_{t}^{c} D_{b}^{\alpha} u$ respectively, are represented by

$$
{ }_{a}^{c} D_{t}^{\alpha} u=\frac{1}{\Gamma(n-\alpha)} \int_{a}^{t}(t-s)^{n-\alpha-1} u^{(n)}(s) d s, t \in[a, b]
$$

and

$$
{ }_{t}^{c} D_{b}^{\alpha} u=\frac{(-1)^{n}}{\Gamma(n-\alpha)} \int_{t}^{b}(t-s)^{n-\alpha-1} u^{(n)}(s) d s, \quad t \in[a, b]
$$

respectively.

(ii) If $\alpha=n-1$ and $u \in A C^{n}\left([a, b], \mathbb{R}^{n}\right)$, then ${ }_{a}^{c} D_{t}^{n-1} u(t)$ and ${ }_{t}^{c} D_{b}^{n-1} u(t)$ are represented by ${ }_{a}^{c} D_{t}^{n-1} u(t)=u^{(n-1)}(t)$ and ${ }_{t}^{c} D_{b}^{n-1} u(t)=(-1)^{n-1} u^{(n-1)}(t)$.

Lemma 1. ([22], Proposition 3.2) Let $0<\alpha \leq 1$, and $1<p<\infty$. For all $u \in L^{p}\left([0, T], \mathbb{R}^{N}\right)$. Then

$$
\left\|{ }_{0} D_{\xi}^{-\alpha} u\right\|_{L^{p}([0, t])} \leq \frac{t^{\alpha}}{\Gamma(\alpha+1)}\|u\|_{L^{p}([0, t])} \text { for } \xi \in[0, t], t \in[0, T],
$$


where ${ }_{0} D_{\xi}^{-\alpha}$ is left Riemann-Liouville fractional integral of order $\alpha$.

Proposition 1. ([24]) We have the following property of fractional integration

$$
\int_{0}^{T}\left[{ }_{0} D_{t}^{-\alpha} u(t)\right] v(t) d t=\int_{0}^{T}\left[{ }_{t} D_{b}^{-\alpha} v(t)\right] u(t) d t, \alpha>0
$$

provided that $u \in L^{p}([0, T], \mathbb{R}), v \in L^{q}([0, T], \mathbb{R})$ and $p \geq 1, \frac{1}{p}+\frac{1}{q} \leq 1+\alpha$ or $p \neq 1, q \neq 1, \frac{1}{p}+\frac{1}{q}=$ $1+\alpha$.

Proposition 2. ([24]) Let $n \in \mathbb{N}$ and $n-1<\alpha \leq n$. If $u \in A C^{n}\left([a, b], \mathbb{R}^{N}\right)$ or $u \in C^{n}\left([a, b], \mathbb{R}^{N}\right)$, then

$$
\begin{gathered}
{ }_{a} D_{t}^{-\alpha}\left({ }_{a}^{c} D_{t}^{\alpha} u(t)\right)=u(t)-\sum_{k=0}^{n-1} \frac{u^{(k)}(a)}{k !}(t-a)^{k} \\
{ }_{t} D_{b}^{-\alpha}\left({ }_{t}^{c} D_{b}^{\alpha} u(t)\right)=u(t)-\sum_{k=0}^{n-1} \frac{(-1)^{k} u^{(k)}(b)}{k !}(b-t)^{k}
\end{gathered}
$$

and for $t \in[a, b]$. In particular, if $0<\alpha \leq 1$ and $u \in A C\left([a, b], \mathbb{R}^{N}\right)$ or $u \in C^{1}\left([a, b], \mathbb{R}^{N}\right)$, then

$$
{ }_{a} D_{t}^{-\alpha}\left({ }_{a}^{c} D_{t}^{\alpha} u(t)\right)=u(t)-u(a) \text {, and }{ }_{t} D_{b}^{-\alpha}\left({ }_{t}^{c} D_{b}^{\alpha} u(t)\right)=u(t)-u(b) .
$$

The Riemann - Liouville fractional derivative and the Caputo fractional derivative are related to one another by the following relationships.

Proposition 3. ([24]) Let $n-1 \leq \alpha<n$.for any $n \in \mathbb{N}$. If $u$ is a function defined on interval $[a, b]$ for which the derivatives of Caputo fractional ${ }_{a}^{c} D_{t}^{\alpha} u(t)$ and ${ }_{t}^{c} D_{b}^{\alpha} u(t)$ of order a exist together with the Riemann-Liouville fractional derivatives ${ }_{a} D_{t}^{\alpha} u(t)$ and ${ }_{t} D_{b}^{\alpha} u(t)$, thus

$$
{ }_{a}^{c} D_{t}^{\alpha} u(t)={ }_{a} D_{t}^{\alpha} u(t)-\sum_{k=0}^{n-1} \frac{u^{(k)}(a)}{\Gamma(k-\alpha+1)}(t-a)^{k-\alpha}
$$

and

$$
{ }_{t}^{c} D_{b}^{\alpha} u(t)={ }_{t} D_{b}^{\alpha} u(t)-\sum_{k=0}^{n-1} \frac{u^{(k)}(b)}{\Gamma(k-\alpha+1)}(b-t)^{k-\alpha},
$$

where $t \in[a, b]$. In particular, when $0<\alpha<1$, we have

$$
{ }_{a}^{c} D_{t}^{\alpha} u(t)={ }_{a} D_{t}^{\alpha} u(t)-\frac{u(a)}{\Gamma(1-\alpha)}(t-a)^{-\alpha}
$$

and

$$
{ }_{t}^{c} D_{b}^{\alpha} u(t)={ }_{t} D_{b}^{\alpha} u(t)-\frac{u(b)}{\Gamma(1-\alpha)}(b-t)^{-\alpha} .
$$

Definition 4. [25] Let $0<\alpha \leq 1$ and $1<p<\infty$. The space of fractional derivative $H_{0}^{\alpha, p}$ is defined by the closure of $C_{0}^{\infty}\left([0, T], \mathbb{R}^{N}\right)$, that is

$$
H_{0}^{\alpha, p}=\overline{C_{0}^{\infty}\left([0, T], \mathbb{R}^{N}\right)}
$$

with respect to the weighted norm

$$
\|u\|_{\alpha, p}=\left(\int_{0}^{T}|u(t)|^{p} d t+\int_{0}^{T}\left|{ }_{0} D_{t}^{\alpha} u(t)\right|^{p} d t\right)^{\frac{1}{p}}
$$


for every $u \in H_{0}^{\alpha, p}$.

Proposition 4. ([25], Proposition 3.1) Let $0<\alpha \leq 1$, and $1<p<\infty$. The fractional derivative space $H_{0}^{\alpha, p}$ is a reflexive and separable Banach space.

Proposition 5. ([25], Proposition 3.2) Let $0<\alpha \leq 1$, and $1<p<\infty$. For all $u \in H_{0}^{\alpha, p}$, we have

$$
\|u\|_{L^{p}} \leq \frac{T^{\alpha}}{\Gamma(\alpha+1)}\left\|_{0}^{c} D_{t}^{\alpha} u(t)\right\|_{L^{p}}
$$

Moreover, if $\alpha>\frac{1}{p}$, then

$$
\|u\|_{\infty} \leq \frac{T^{\alpha-\frac{1}{p}}}{\Gamma(\alpha)((\alpha-1) q+1)^{\frac{1}{q}}}\left\|_{0}^{c} D_{t}^{\alpha} u(t)\right\|_{L^{p}}
$$

Proposition 6. ([25], Proposition 3.3) Let $0<\alpha \leq 1$ and $1<p<\infty$. Assume that $\alpha>\frac{1}{p}$ and the sequence $\left\{u_{k}\right\}$ converges weakly to $u$ in $H_{0}^{\alpha, p}$, i.e., $u_{k} \rightarrow$ u. Then $u_{k} \rightarrow u$ in $C_{0}^{\infty}\left([0, T], \mathbb{R}^{N}\right)$, i.e., $\left\|u-u_{k}\right\|_{\infty} \rightarrow 0$, as $k \rightarrow \infty$.

In our study in this paper, we use Hilbert space $H^{\alpha}=H_{0}^{\alpha, 2}$ with the inner product and the following corresponding norm

$$
\langle u, v\rangle=\int_{0}^{T}(u(t), v(t)) d t+\int_{0}^{T}\left({ }_{0}^{c} D_{t}^{\alpha} u(t){ }_{0}^{c} D_{t}^{\alpha} v(t)\right) d t, \forall u, v \in H^{\alpha}
$$

and

$$
\|u\|_{\alpha}=\|u\|_{\alpha, 2}=\left(\int_{0}^{T}|u(t)|^{2} d t+\int_{0}^{T}\left|{ }_{0}^{c} D_{t}^{\alpha} u(t)\right|^{2} d t\right)^{\frac{1}{2}}, \forall u \in H^{\alpha}
$$

In view of (11), we have that, for $t \in[0, T]$ and $p=q=2$

$$
\|u\|_{\infty} \leq \xi\left\|_{0}^{c} D_{t}^{\alpha} u\right\|_{L^{2}} \leq \xi\|u\|_{\alpha}
$$

Definition 5. Let $X$ be a Banach space with $J: X \rightarrow \mathbb{R}$ differentiable. It can be said that $J$ satisfies the Palais-Smale (PS)-condition if for all sequence $\left(u_{n}\right)$ in $X$ which $J\left(u_{n}\right)$ is bounded and $J^{\prime}\left(u_{n}\right) \rightarrow 0$ as $n \rightarrow \infty$ possesses a convergent subsequence.

Definition 6. Let $X$ be a Banach space and $J: X \rightarrow \mathbb{R}$ differentiable and $c \in \mathbb{R}$. It can be said that $J$ satisfies the $(P S)_{c}$-condition if the existence of a sequence $\left(u_{n}\right)$ in $X$ such that

$$
J\left(u_{n}\right) \rightarrow c, \quad J^{\prime}\left(u_{n}\right) \rightarrow 0
$$

as $n \rightarrow \infty$, implies that $c$ is a critical value of $J$.

Remark 1. It can be remarked the (PS)-condition implies the $(P S)_{c}$ condition for each $c \in R$.

Lemma 2. [23] Let $X$ be a Banach space with a direct sum decomposition $X=X_{1} \oplus X_{2}$ and $k:=\operatorname{dim} X_{2}<\infty$. Let $J \in C^{1}(X, \mathbb{R})$ with $J(0)=0$, satisfying the $(P S)$-condition, we assume that, for $\rho>0$, 


$$
\begin{aligned}
& J(u) \leq 0, u \in X_{2},\|u\|_{\alpha} \leq \rho, \\
& J(u) \geq 0, u \in X_{1},\|u\|_{\alpha} \leq \rho
\end{aligned}
$$

In addition, we assume also that $J$ is bounded below and $\inf _{X} J<0$. Thus $J$ has at least two non zero critical points.

\section{Mains Result}

We establish the existence of at least two non zero weak solutions to problem (1) via Brezis and Nirenberg's Linking Theorem.

Definition 7. A function $u \in H^{\alpha}$ is said to be a weak solution of problem (1) if the identity

$$
\int_{0}^{T}\left({ }_{0}^{c} D_{t}^{\alpha} u(t)_{0}^{c} D_{t}^{\alpha} v(t)\right) d t+\frac{1}{2} \sum_{i=1}^{N} \sum_{j=1}^{L} I_{i j}\left(u^{i}\left(t_{j}\right)\right) v^{i}\left(t_{j}\right) d t+\int_{0}^{T}(\nabla F(t, u(t)), v(t)) d t=0
$$

holds for any $v \in H^{\alpha}$.

Consider the functional $J: H^{\alpha} \rightarrow \mathbb{R}$ defined by

$$
\begin{aligned}
& J(u)=\frac{1}{2} \int_{0}^{T}\left|{ }_{0}^{c} D_{t}^{\alpha} u(t)\right|^{2} d t+\int_{0}^{T} F(t, u(t)) d t+\frac{1}{2} \sum_{i=1}^{N} \sum_{j=1}^{L} \int_{0}^{u^{i}\left(t_{j}\right)} I_{i j}(s) d s \\
& =J_{1}(u)+J_{2}(u)
\end{aligned}
$$

where

$$
J_{1}(u)=\frac{1}{2} \int_{0}^{T}\left|{ }_{0}^{c} D_{t}^{\alpha} u(t)\right|^{2} d t+\int_{0}^{T} F(t, u(t)) d t
$$

and

$$
J_{2}(u)=\frac{1}{2} \sum_{i=1}^{N} \sum_{j=1}^{L} \int_{0}^{u^{i}\left(t_{j}\right)} I_{i j}(s) d t
$$

Proposition 7. The functional $J$ is continuously differentiable on $H^{\alpha}$ and

$$
\left\langle J^{\prime}(u), v\right\rangle=\int_{0}^{T}{ }_{0}^{c} D_{t}^{\alpha} u(t)_{0}^{c} D_{t}^{\alpha} v(t) d t+\frac{1}{2} \sum_{i=1}^{N} \sum_{j=1}^{L} I_{i j}\left(u^{i}\left(t_{j}\right)\right) v^{i}\left(t_{j}\right) d t+\int_{0}^{T}(\nabla F(t, u(t)), v(t)) d t
$$

Proof. The proof of this proposition is very simple, we omit it.

It is clear that, the critical points of $J$ are weak solutions of (1). Now, we give the proof of our main results.

Proof of Theorem 1. We apply Lemma 1 to $J$. Knowing that $H^{\alpha}$ is a Banach space and $J \in C^{1}\left(H^{\alpha}, \mathbb{R}\right)$ (see Proposition 5). By (15), it can be easily checked that functional $J$ satisfies $J(0)=0$. We decompose the proof of the theorem into the following three steps.

Step1 We get $(h 1)$ or $(h 2)$ implies that

$$
\lim _{\|u\|_{\alpha} \rightarrow \infty} J(u)=+\infty
$$


and $J(u)$ is bounded below on $H^{\alpha}$. To this end, for any $0<\varepsilon<\gamma,(h 1)$ implies that there exists $\delta>0$ such that

$$
F(t, u(t)) \geq(\gamma-\varepsilon)|u|^{2}, \quad \forall u \in \mathbb{R}^{N} \text { with }|u| \geq \delta \text { and a.e.t } \in[0, T] .
$$

Let $a_{\delta}=\max _{|u| \leq \delta} a(|u|)$; in view of (A), one can get

$$
\begin{aligned}
& F(t, u) \geq-a(|u|) b(t) \geq-a_{\delta} b(t)+(\gamma-\varepsilon)|u|^{2}-(\gamma-\varepsilon) \delta^{2}, \\
& \forall u \in \mathbb{R}^{N} \text { with }|u| \geq \delta \text { and a.e.t } \in[0, T] .
\end{aligned}
$$

Then it follows from (20) and (21) that

$$
F(t, u) \geq(\gamma-\varepsilon)|u|^{2}-(\gamma-\varepsilon) \delta^{2}-a_{\delta} b(t), \forall u \in \mathbb{R}^{N} \text { with }|u| \geq \delta \text { and a.e.t } \in[0, T] .
$$

Thus, by (16) and (22), we have

$$
\begin{aligned}
& J_{1}(u)=\frac{1}{2} \int_{0}^{T}\left|{ }_{0}^{c} D_{t}^{\alpha} u(t)\right|^{2} d t+\int_{0}^{T} F(t, u(t)) d t \\
& \geq \frac{1}{2} \int_{0}^{T}\left|{ }_{0}^{c} D_{t}^{\alpha} u(t)\right|^{2} d t+(\gamma-\varepsilon) \int_{0}^{T}|u(t)|^{2} d t \\
& -(\gamma-\varepsilon) \delta^{2} T-a_{\delta} \int_{0}^{T} b(t) d t \\
& \geq \min \left\{\frac{1}{2},(\gamma-\varepsilon)\right\}\|u\|_{\alpha}^{2} \\
& -(\gamma-\varepsilon) \delta^{2} T-a_{\delta} \int_{0}^{T} b(t) d t
\end{aligned}
$$

for all $u \in H_{0}^{\alpha}$. For any $z \in \mathbb{R}$, we set

$$
K(\tau):=\left(\int_{0}^{\tau^{-1} z} I_{i j}(s) d s\right) \tau^{\gamma_{i j}}, \text { for all } \tau \geq 1 .
$$

By $(h 3)$

$$
\begin{aligned}
K^{\prime}(\tau) & :=-\tau^{\gamma_{i j}-2} z I_{i j}\left(\tau^{-1} z\right)+\gamma_{i j}\left(\int_{0}^{\tau^{-1} z} I_{i j}(s) d s\right) \tau^{\gamma_{i j}-1} \\
& =\tau^{\gamma_{i j}-1}\left(\gamma_{i j}\left(\int_{0}^{\tau^{-1} z} I_{i j}(s) d s\right)-\tau^{-1} z I_{i j}\left(\tau^{-1} z\right)\right) \leq 0
\end{aligned}
$$

so that $K$ is non-increasing in $[1, \infty)$. Therefore, for any $|z| \geq 1$, we have $K(1) \geq K(|z|)$, that is,

$$
\int_{0}^{z} I_{i j}(s) d s \geq\left(\int_{0}^{|z|^{-1} z} I_{i j}(s) d s\right)|z|^{\gamma_{i j}} \geq c_{i j}|z|^{\gamma_{i j}}
$$

where $c_{i j}=\inf _{|z|=1} \int_{0}^{z} I_{i j}(s) d s>0$ by (h4) Thus, it follows from (15), (16), (18), (23) and (24) that 


$$
\begin{aligned}
& J(u) \geq \min \left\{\frac{1}{2},(\gamma-\varepsilon)\right\}\|u\|_{\alpha}^{2}-(\gamma-\varepsilon) \delta^{2} T-a_{\delta} \int_{0}^{T} b(t) d t+\frac{1}{2} \sum_{i=1}^{N} \sum_{j=1}^{L} \int_{0}^{u^{i}\left(t_{j}\right)} I_{i j}(s) d t \\
& \geq \min \left\{\frac{1}{2},(\gamma-\varepsilon)\right\}\|u\|_{\alpha}^{2}-(\gamma-\varepsilon) \delta^{2} T-a_{\delta} \int_{0}^{T} b(t) d t-\frac{1}{2} \sum_{i=1}^{N} \sum_{j=1}^{L} c_{i j}\left|u^{i}\left(t_{j}\right)\right|^{\gamma_{i j}} \\
& \geq \min \left\{\frac{1}{2},(\gamma-\varepsilon)\right\}\|u\|_{\alpha}^{2}-\frac{1}{2} \sum_{i=1}^{N} \sum_{j=1}^{L} c_{i j}\left\|u^{i}\left(t_{j}\right)\right\|_{\infty}^{\gamma_{i j}}-(\gamma-\varepsilon) \delta^{2} T-a_{\delta} \int_{0}^{T} b(t) d t \\
& \geq \min \left\{\frac{1}{2},(\gamma-\varepsilon)\right\}\|u\|_{\alpha}^{2}-\frac{1}{2} \sum_{i=1}^{N} \sum_{j=1}^{L} c_{i j} \xi^{\gamma} \gamma_{i j}\left\|u^{i}\left(t_{j}\right)\right\|_{\alpha}^{\gamma_{i j}}-(\gamma-\varepsilon) \delta^{2} T-a_{\delta} \int_{0}^{T} b(t) d t
\end{aligned}
$$

Then in view of (25), for any $\gamma>0$, choosing $0<\varepsilon<\gamma$. The following two cases may occur.

Case1 $\gamma>\frac{1}{2}$ choosing $\varepsilon=\frac{\gamma-\frac{1}{2}}{2}$, we can get $\gamma-\varepsilon>\frac{1}{2}$, so

$$
\min \left\{\frac{1}{2},(\gamma-\varepsilon)\right\}=\frac{1}{2}
$$

Thus, it follows from (25) that

$$
J(u) \geq \frac{1}{2}\|u\|_{\alpha}^{2}-\frac{1}{2} \sum_{i=1}^{N} \sum_{j=1}^{L} c_{i j} \xi^{\gamma_{i j}}\left\|u^{i}\left(t_{j}\right)\right\|_{\alpha}^{\gamma_{i j}}-\frac{1}{2}\left(\gamma+\frac{1}{2}\right) \delta^{2} T-a_{\delta} \int_{0}^{T} b(t) d t .
$$

So (H1) yields that (19) holds, i.e.,

$$
J(u) \rightarrow \infty \text {, as }\|u\|_{\alpha} \rightarrow \infty
$$

Case $2 \gamma \leq \frac{1}{2}$ choosing $\varepsilon=\frac{\gamma-\xi^{2} \sum_{i=1}^{N} \sum_{j=1}^{L} \frac{{ }^{c} i j}{2}}{2}$ It follows from (H2) that

$$
\gamma-\varepsilon=\frac{1}{2}\left(\gamma+\frac{1}{2} \xi^{2} \sum_{i=1}^{N} \sum_{j=1}^{L} c_{i j}\right)>\frac{1}{2} \xi^{2} \sum_{i=1}^{N} \sum_{j=1}^{L} c_{i j}>0
$$

and $\varepsilon>0$. Since $\gamma-\varepsilon \leq \frac{1}{2}-\varepsilon<\frac{1}{2}$, we have

$$
\min \left\{\frac{1}{2},(\gamma-\varepsilon)\right\}=(\gamma-\varepsilon) .
$$

Thus, it follows from (19) and (20) that

$$
\begin{aligned}
J(u) \geq & (\gamma-\varepsilon)\|u\|_{\alpha}^{2}-\frac{1}{2} \sum_{i=1}^{N} \sum_{j=1}^{L} c_{i j} \xi^{\gamma_{i j}}\left\|u^{i}\left(t_{j}\right)\right\|_{\alpha}^{\gamma_{i j}}-(\gamma-\varepsilon) \delta^{2} T-a_{\delta} \int_{0}^{T} b(t) d t \\
\geq & (\gamma-\varepsilon)\|u\|_{\alpha}^{2}-\frac{1}{2} \sum_{i=1}^{N} \sum_{j=1}^{L} c_{i j} \xi^{\gamma_{i j}}\left\|u^{i}\left(t_{j}\right)\right\|_{\alpha}^{\gamma_{i j}}-\frac{1}{2}\left(\gamma+\frac{1}{2} \xi^{2} \sum_{i=1}^{N} \sum_{j=1}^{L} c_{i j}\right) \delta^{2} T \\
& -a_{\delta} \int_{0}^{T} b(t) d t
\end{aligned}
$$

which combined with (H2) yields that (19) holds, i.e.,

$$
J(u) \rightarrow \infty, \text { as }\|u\|_{\alpha} \rightarrow \infty \text {. }
$$

Therefore, $(H 1)$ or $(H 2)$ implies (19). Thus, $J(u)$ is bounded below on $H^{\alpha}$. 
Step 2 We prove that $(H 1)$ or $(H 2)$ implies that $J(u)$ satisfies the $(P S)$ condition. Suppose that $\left\{u_{n}\right\}$ is a sequence in $H^{\alpha}$ such that $J\left(u_{n}\right)$ is bounded and $J^{\prime}\left(u_{n}\right) \rightarrow 0$ as $n \rightarrow \infty$. Then $\left\{u_{n}\right\}$ is bounded on $H_{0}^{\alpha}$. In fact, if $\left\{u_{n}\right\}$ is an unbounded sequence, without loss of generality we assume that $\left\|u_{n}\right\|_{\alpha} \rightarrow \infty$ as $n \rightarrow \infty$. By Step 1, we know that (H1) or (H2) implies (19). Thus $J\left(u_{n}\right) \rightarrow \infty$, which contradicts the boundedness of $J\left(u_{n}\right)$. Since $\left\{u_{n}\right\} \subset H^{\alpha}$ is bounded and $H^{\alpha}$ is a reflexive Banach space and so by passing to a subsequence (for simplicity denoted again by $\left\{u_{n}\right\}$ ) if necessary, by Proposition 6, we may assume that

$$
\left\{\begin{array}{l}
u_{n} \rightarrow u, \text { weakly in } H^{\alpha} \\
u_{n} \rightarrow u, \text { strongly in } C\left([0, T], \mathbb{R}^{N}\right)
\end{array}\right.
$$

By (18), we have

$$
\begin{aligned}
\left\langle J^{\prime}\left(u_{n}\right)-J^{\prime}(u), u_{n}-u\right\rangle= & \int_{0}^{T}\left|{ }_{0}^{c} D_{t}^{\alpha} u_{n}(t)-{ }_{0}^{c} D_{t}^{\alpha} u(t)\right|^{2} d t \\
& +\frac{1}{2} \sum_{i=1}^{N} \sum_{j=1}^{L}\left[I_{i j}\left(u_{n}^{i}\left(t_{j}\right)\right)-I_{i j}\left(u^{i}\left(t_{j}\right)\right)\right]\left(u_{n}^{i}\left(t_{j}\right)-u^{i}\left(t_{j}\right)\right) d t \\
& +\int_{0}^{T}\left(\nabla F\left(t, u_{n}(t)-\nabla F(t, u(t))\right), u_{n}(t)-u(t)\right) d t \\
\geq & \int_{0}^{T}\left|{ }_{0}^{c} D_{t}^{\alpha} u_{n}(t)-{ }_{0}^{c} D_{t}^{\alpha} u(t)\right|^{2} d t \\
& +\frac{1}{2} \sum_{i=1}^{N} \sum_{j=1}^{L}\left[I_{i j}\left(u_{n}^{i}\left(t_{j}\right)\right)-I_{i j}\left(u^{i}\left(t_{j}\right)\right)\right]\left(u_{n}^{i}\left(t_{j}\right)-u^{i}\left(t_{j}\right)\right) d t \\
& +\int_{0}^{T}\left(\nabla F\left(t, u_{n}(t)-\nabla F(t, u(t))\right), u_{n}(t)-u(t)\right) d t
\end{aligned}
$$

One has

$$
\begin{aligned}
& \int_{0}^{T}\left|{ }_{0}^{c} D_{t}^{\alpha} u_{n}(t)-{ }_{0}^{c} D_{t}^{\alpha} u(t)\right|^{2} d t \leq\left\langle J^{\prime}\left(u_{n}\right)-J^{\prime}(u), u_{n}-u\right\rangle \\
& -\frac{1}{2} \sum_{i=1}^{N} \sum_{j=1}^{L}\left[I_{i j}\left(u_{n}^{i}\left(t_{j}\right)\right)-I_{i j}\left(u^{i}\left(t_{j}\right)\right)\right]\left(u_{n}^{i}\left(t_{j}\right)-u^{i}\left(t_{j}\right)\right) d t \\
& -\int_{0}^{T}\left(\nabla F\left(t, u_{n}(t)-\nabla F(t, u(t))\right), u_{n}(t)-u(t)\right) d t
\end{aligned}
$$

By (27), we know that

$$
\int_{0}^{T}\left|u_{n}(t)-u(t)\right|^{2} d t \rightarrow 0, \text { as } n \rightarrow \infty
$$

and for any $i \in A, j \in B$, we have that $u_{n}^{i}\left(t_{j}\right) \rightarrow u^{i}\left(t_{j}\right)$, as $n \rightarrow \infty$. In fact

$$
\left|u_{n}^{i}\left(t_{j}\right)-u^{i}\left(t_{j}\right)\right| \leq\left|u_{n}\left(t_{j}\right)-u\left(t_{j}\right)\right| \quad \text { for any } i \in A, j \in B,
$$

Thus, it follows from the continuity of all $I_{i j}$ that

$$
\sum_{i=1}^{N} \sum_{j=1}^{L}\left[I_{i j}\left(u_{n}^{i}\left(t_{j}\right)\right)-I_{i j}\left(u^{i}\left(t_{j}\right)\right)\right]\left(u_{n}^{i}\left(t_{j}\right)-u^{i}\left(t_{j}\right)\right) \rightarrow 0, \text { as } n \rightarrow \infty .
$$


In view of (A) and (27), we have

$$
\int_{0}^{T}\left(\nabla F\left(t, u_{n}(t)-\nabla F(t, u(t))\right), u_{n}(t)-u(t)\right) d t \rightarrow 0, \text { as } n \rightarrow \infty .
$$

Since $J^{\prime}\left(u_{n}\right) \rightarrow 0$, then using (28), (30) and (31), we have that

$$
\int_{0}^{T}\left|{ }_{0}^{c} D_{t}^{\alpha} u_{n}(t)-{ }_{0}^{c} D_{t}^{\alpha} u(t)\right|^{2} d t \rightarrow 0, \text { as } n \rightarrow \infty .
$$

By (29) and (32), one has $\left\|u_{n}-u\right\|_{\alpha} \rightarrow 0$, which means that $J(u)$ satisfies the (PS)-condition.

Step 3 We prove that (H3) implies that (14) holds for some $\rho>0$. To this end, it follows from (13), (17) and (h5) , that

$$
\begin{aligned}
0 \leq & J_{2}(u)=\frac{1}{2} \sum_{i=1}^{N} \sum_{j=1}^{L} \int_{0}^{u^{i}\left(t_{j}\right)} I_{i j}(s) d t \\
\leq & \frac{1}{2} \sum_{i=1}^{N} \sum_{j=1}^{L} k^{2} \omega^{2} \mu_{i j}\left|u^{i}\left(t_{j}\right)\right|^{2} \\
\leq & \frac{1}{2} \sum_{i=1}^{N} \sum_{j=1}^{L} k^{2} \omega^{2} \mu\|u\|_{\infty}^{2} \\
\leq & \frac{1}{2} \sum_{i=1}^{N} \sum_{j=1}^{L} k^{2} \omega^{2} \mu \xi^{2}\|u\|_{\alpha}^{2} \\
& =\frac{1}{2} N L k^{2} \omega^{2} \mu \xi^{2}\|u\|_{\alpha}^{2}
\end{aligned}
$$

where $\mu=\max _{i \in A, j \in B}\left\{\mu_{i j}\right\}$.Let

$$
X_{2}=\left\{\sum_{m=0}^{k} a_{m} \sin (m \omega t) ; a_{m} \in \mathbb{R}^{N}\right\}
$$

where $a_{m}=\left(a_{m}^{1}, a_{m}^{2}, \ldots, a_{m}^{N}\right)$ with $a_{m}^{i}=1$ for $i=1, \ldots, N$. and $X_{1}$ be the orthogonal complement of $X_{2}$ in $H^{\alpha}$. Then $\operatorname{dim} X_{2}<\infty$ and $H^{\alpha}=X_{1} \oplus X_{2}$.

If (H3) holds, we will consider $X_{2}$ with $k \geq 1$. When $u \in X_{2}$, we have

$$
\begin{aligned}
& \int_{0}^{T}|u(t)|^{2} d t=\int_{0}^{T} \sum_{i=1}^{N}\left|u^{i}(t)\right|^{2} d t=\int_{0}^{T} \sum_{i=1}^{N}\left(\sum_{m=0}^{k} a_{m}^{i} \sin (m \omega t)\right)^{2} d t \\
& =\int_{0}^{T} \sum_{i=1}^{N}\left(\sum_{m=0}^{k}\left(a_{m}^{i}\right)^{2} \sin ^{2}(m \omega t)+\sum_{m=0}^{k} \sum_{j=0, j \neq m}^{k} a_{m}^{i} a_{j}^{i} \sin (m \omega t) \sin (j \omega t)\right) d t
\end{aligned}
$$

On the other hand, one has

$$
\int_{0}^{T} \sin (m \omega t) \sin (j \omega t) d t=\left\{\begin{array}{l}
\frac{T}{2}, m=j \\
0, \text { other wise. }
\end{array}\right.
$$


So, in view of (34) and (35), we have

$$
\int_{0}^{T}|u(t)|^{2} d t=\frac{T}{2} \sum_{i=1}^{N} \sum_{m=0}^{k}\left(a_{m}^{i}\right)^{2}=\frac{N(k+1) T}{2}
$$

Also, For all $u \in X_{2}$, and by Proposition 3, one has ${ }_{0}^{c} D_{t}^{\alpha} u(t)={ }_{0} D_{t}^{\alpha} u(t)$ and

$$
\begin{aligned}
& { }_{0}^{c} D_{t}^{\alpha} u^{i}(t)={ }_{0}^{c} D_{t}^{\alpha} u^{i}(t)=\frac{1}{\Gamma(1-\alpha)} \frac{d}{d t}\left(\int_{0}^{T}(t-s)^{-\alpha} \sum_{m=0}^{k} a_{m}^{i} \sin (m \omega s) d s\right) \\
& \leq \frac{1}{\Gamma(1-\alpha)} \frac{d}{d t}\left(\int_{0}^{T}(t-s)^{-\alpha} \sum_{m=0}^{k} a_{m}^{i}(m \omega s) d s\right) \\
& =\frac{1}{\Gamma(1-\alpha)} \frac{d}{d t} \sum_{m=0}^{k} a_{m}^{i}(m \omega)\left(\frac{t^{2-\alpha}}{(1-\alpha)(2-\alpha)}\right) \\
& =\frac{1}{(1-\alpha) \Gamma(1-\alpha)} \sum_{m=0}^{k} a_{m}^{i}(m \omega) t^{1-\alpha} \\
& =\frac{1}{\Gamma(2-\alpha)} \sum_{m=0}^{k} a_{m}^{i}(m \omega) t^{1-\alpha}
\end{aligned}
$$

which implies

$$
\begin{aligned}
& \int_{0}^{T}\left|{ }_{0}^{c} D_{t}^{\alpha} u(t)\right|^{2} d t \leq\left(\frac{1}{\Gamma(2-\alpha)}\right)^{2} \int_{0}^{T} \sum_{i=1}^{N}\left(\sum_{m=0}^{k}\left(a_{m}^{i}\right)^{2} m^{2} \omega^{2}+\sum_{m=0}^{k} \sum_{j=0, j \neq m}^{k} m j \omega^{2} a_{m}^{i} a_{j}^{i}\right) t^{2(1-\alpha)} d t \\
& =\left(\frac{1}{\Gamma(2-\alpha)}\right)^{2} \sum_{i=1}^{N}\left(\sum_{m=0}^{k}\left(a_{m}^{i}\right)^{2} m^{2} \omega^{2}+\sum_{m=0}^{k} \sum_{j=0, j \neq m}^{k} m j \omega^{2} a_{m}^{i} a_{j}^{i}\right) \frac{T^{3-2 \alpha}}{3-2 \alpha} \\
& \leq\left(\frac{1}{\Gamma(2-\alpha)}\right)^{2}(2 k+1) k^{2} \omega^{2} \frac{T^{3-2 \alpha}}{3-2 \alpha}
\end{aligned}
$$

By (16) and (h2), for all $\|u\|_{\alpha} \leq \rho$, we have

$$
\begin{aligned}
J_{1}(u) & =\frac{1}{2} \int_{0}^{T}\left|{ }_{0}^{c} D_{t}^{\alpha} u(t)\right|^{2} d t+\int_{0}^{T} F(t, u(t)) d t \\
& \leq \frac{1}{2} \int_{0}^{T}\left|{ }_{0}^{c} D_{t}^{\alpha} u(t)\right|^{2} d t-\frac{1}{2} \lambda k^{2} \omega^{2}\left(\frac{1}{\Gamma(2-\alpha)}\right)^{2} \int_{0}^{T}|u(t)|^{2} d t .
\end{aligned}
$$

By (15), (33), (36)-(38), we have

$$
\begin{aligned}
& J(u) \leq \frac{1}{2} \int_{0}^{T}\left|{ }_{0}^{c} D_{t}^{\alpha} u(t)\right|^{2} d t-\frac{1}{2} \lambda k^{2} \omega^{2}\left(\frac{1}{\Gamma(2-\alpha)}\right)^{2} \int_{0}^{T}|u(t)|^{2} d t \\
& +\frac{1}{2} N L k^{2} \omega^{2} \mu \xi^{2}\|u\|_{\alpha}^{2}, \\
& \leq \frac{1}{2}\left(\frac{1}{\Gamma(2-\alpha)}\right)^{2}(2 k+1) k^{2} \omega^{2} \frac{T^{3-2 \alpha}}{3-2 \alpha}-\frac{1}{4} \lambda k^{2} \omega^{2} N(k+1) T\left(\frac{1}{\Gamma(2-\alpha)}\right)^{2} \\
& +\frac{1}{2} N L k^{2} \omega^{2} \mu \xi^{2}\|u\|_{\alpha}^{2},
\end{aligned}
$$

for all $u \in X_{2}$. Therefore, by the fact $1<\left(\frac{1}{\Gamma(2-\alpha)}\right)^{2}$ for $\alpha \in\left(\frac{1}{2}, 1\right], 2 k+1<2 k+2, N>1$, (36), (37) and (39), we have 


$$
\begin{aligned}
J(u) \leq & \left(\frac{1}{\Gamma(2-\alpha)}\right)^{2}(2 k+1) k^{2} \omega^{2} \frac{T^{3-2 \alpha}}{3-2 \alpha} \\
& -\frac{1}{4} \lambda k^{2} \omega^{2} N(k+1) T\left(\frac{1}{\Gamma(2-\alpha)}\right)^{2} \\
& +\frac{1}{4} L N^{2}(k+1) k^{2} \omega^{2} \xi^{2} T \\
& +\left(\frac{1}{\Gamma(2-\alpha)}\right) L N(2 k+1) k^{4} \omega^{4} \mu \xi^{2} \frac{T^{3-2 \alpha}}{3-2 \alpha} \\
\leq & \left(\frac{1}{\Gamma(2-\alpha)}\right)^{2}(2 k+2) k^{2} \omega^{2} \frac{T^{3-2 \alpha}}{3-2 \alpha} \\
& -\frac{1}{4} \lambda k^{2} \omega^{2} N(k+1) T\left(\frac{1}{\Gamma(2-\alpha)}\right)^{2} \\
& +\frac{1}{4} L N^{2}(k+1) k^{2} \omega^{2} \mu \xi^{2} T\left(\frac{1}{\Gamma(2-\alpha)}\right)^{2} \\
& +\left(\frac{1}{\Gamma(2-\alpha)}\right)^{2} L N(2 k+2) k^{4} \omega^{4} \mu \xi^{2} \frac{T^{3-2 \alpha}}{3-2 \alpha} \\
\leq & \frac{2 k^{2}(k+1) \pi^{2}}{T}\left(\frac{1}{\Gamma(2-\alpha)}\right)^{2} \\
& \times\left[2 \frac{T^{2-2 \alpha}}{3-2 \alpha}-\frac{1}{2} \lambda N+\frac{1}{2} L N^{2} \mu \xi^{2}+2 L N k^{2} \omega^{2} \mu \xi^{2} \frac{T^{2-2 \alpha}}{3-2 \alpha}\right]
\end{aligned}
$$

Thus

$$
\begin{aligned}
& J(u) \leq \frac{k^{2}(k+1) N \pi^{2}}{T}\left(\frac{1}{\Gamma(2-\alpha)}\right)^{2} \\
& \times\left[4 \frac{T^{2-2 \alpha}}{3-2 \alpha}-\lambda+L N \mu \xi^{2}+4 L k^{2} \omega^{2} \mu \xi^{2} \frac{T^{2-2 \alpha}}{3-2 \alpha}\right]
\end{aligned}
$$

for all $u \in X_{2}$ with $\|u\|_{\alpha} \leq \rho$. Thus, (H3) implies that $J(u) \leq 0$ for all $u \in X_{2}$ with $\|u\|_{\alpha} \leq \rho$.

In view of (22) by choosing $\varepsilon=\frac{\gamma}{2}$, we get

$$
\begin{aligned}
& J_{1}(u)= \frac{1}{2} \int_{0}^{T}\left|{ }_{0}^{c} D_{t}^{\alpha} u(t)\right|^{2} d t+\int_{0}^{T} F(t, u(t)) d t \\
& \geq \frac{1}{2} \int_{0}^{T}\left|{ }_{0}^{c} D_{t}^{\alpha} u(t)\right|^{2} d t+\frac{\gamma}{2} \int_{0}^{T}|u(t)|^{2} d t \\
&-\frac{\gamma}{2} \delta^{2} T-a_{\delta} \int_{0}^{T} b(t) d t \\
& \geq \min \left\{\frac{1}{2}, \frac{\gamma}{2}\right\}\|u\|_{\alpha}^{2}-\frac{\gamma}{2} \delta^{2} T-a_{\delta} \int_{0}^{T} b(t) d t,
\end{aligned}
$$

for all $u \in H^{\alpha}$. Since $b \in L^{1}\left([0, T], \mathbb{R}^{+}\right)$, then $J(u) \geq 0$ for all $u \in X_{1}$ with $\|u\|_{\alpha} \leq \rho$ for $\rho$ large enough. Thus, (H3) implies that (14) holds for $\rho$ large enough.

Moreover, it follows from $(h 2)$ that $F(t, 0)=0$ for a.e. $t \in[0, T]$.Thus, $(h 2)$ implies $J(0)=0$. Now if $\inf _{H^{\alpha}} J \geq 0$, by Step 3 we have that all $u \in X_{2}$ with $\|u\|_{\alpha} \leq \rho$ are minima of $J$, which implies that $J$ has infinitely many critical points. If $\inf _{H^{\alpha}} J \leq 0$, then it follows from Lemma 2 that $J$ has at least two nonzero critical points. Therefore, problem (1) has at least two nonzero weak solutions in $H^{\alpha}$. The proof of Theorem 1 is complete. 
Example 1. Let $\alpha=\frac{3}{4}, T=2 \pi, N=1$ and $k=1$. Consider the boundary value problem of the fractional differential equation with impulsive effects

$$
\left\{\begin{array}{l}
{ }_{t} D_{T}^{\frac{3}{4}}\left({ }_{0}^{c} D_{t}^{\frac{3}{4}} u(t)\right)=-\nabla F(t, u), t \in[0,2 \pi] \\
\triangle\left({ }_{t} D_{T}^{\frac{-1}{4}}\left({ }_{0} D_{T}^{\frac{3}{4}} u^{i}\right)\left(t_{j}\right)\right)=\frac{1}{2} I_{i j}\left(u^{i}\left(t_{j}\right)\right), \quad i=1, j=1 \\
u(0)=u(2 \pi)=0
\end{array}\right.
$$

where $I_{i j}(s)=\frac{1}{5}(0.0005)$ s., Direct computation shows that $\xi=\frac{(2 \pi)^{\frac{1}{4}} \sqrt{2}}{\Gamma\left(\frac{3}{4}\right)} \approx 1.8, \omega=1,(h 3),(h 4)$ and $(h 5)$ hold with $\gamma_{i j}=\frac{1}{2}$ and $\mu=\frac{1}{2000}$.

Case 1 Assume that $F$ of $(42)$ is

$$
F(t, u)=\left\{\begin{array}{l}
\frac{1}{2}(t+2)\left(|u|^{2}-|u|\right), \quad|u|>1, \\
5.71187(t+1)\left(|u|^{4}-|u|^{2}\right), \quad|u| \leq 1,
\end{array}\right.
$$

where $t \in[0,2 \pi]$. In this case, we have that condition $(A)$ holds with $a(|u|)=$ $\max \left\{|u|^{4}+|u|^{2},\left(2|u|^{2}+2|u|\right), 4|u|^{3}+2|u|, 4|u|+2\right\}$ and $b(t)=7(t+2)$. Direct computation shows that ( $h 1$ ) holds with $\gamma=1$ since, we have

$$
\lim _{|u| \rightarrow \infty} \inf \frac{F(t, u)}{|u|^{2}}=\frac{1}{2}(t+2) \geq \gamma>\frac{1}{2}
$$

Moreover, direct computation shows that (h2) holds with $\lambda=9.46$ and

$$
\lambda \geq 9.45+24.89 \mu \approx 9.46,
$$

where $\Gamma(2-\alpha)=\Gamma\left(\frac{5}{4}\right) \approx 0.91$. Since $\lim _{|u| \rightarrow 0} \frac{F(t, u)}{|u|^{2}}=-5.71187(t+1)$, then by choosing $\varepsilon=\frac{1}{100}$ such that $F(t, u) \leq-5.71187|u|^{2}$.

Thus, by $F(t, 0)=0,(H 1)$ and (H3) holds. According to Theorem 1, the problem (42) has at least two non zero weak solutions.

Case 2 Assume that $F$ of (42) is

$$
F(t, u)=\left\{\begin{array}{l}
\left(t+\frac{1}{3}\right)\left(|u|^{2}-|u|\right), \quad|u|>1, \\
9.77(t+0.605)\left(|u|^{4}-|u|^{2}\right), \quad|u| \leq 1
\end{array}\right.
$$

where $t \in[0,2 \pi]$. In this case, we have that condition (A) holds with $a(|u|)=$ $\max \left\{|u|^{4}+|u|^{2},\left(2|u|^{2}+2|u|\right), 4|u|^{3}+2|u|, 4|u|+2\right\}$ and $b(t)=10(t+1)$. Direct computation shows that $(h 1)$ holds with $\gamma=\frac{1}{3}$, since

$$
\lim _{|u| \rightarrow \infty} \inf \frac{F(t, u)}{|u|^{2}}=t+\frac{1}{3} \geq \gamma,
$$


and

$$
0.06593 \approx \xi^{2} \sum_{i}^{1} \sum_{j}^{1} \frac{c_{i j}}{2}<\gamma \leq \frac{1}{2} .
$$

Moreover, direct computation shows that (h2) holds. Since

$$
\lim _{|u| \rightarrow 0} \frac{F(t, u)}{|u|^{2}}=-9.77(t+0.605) .
$$

Therefore, by choosing $\varepsilon=\frac{132}{1000}$, we have $F(t, u) \leq-5.71187|u|^{2}$.Then, by $F(t, 0)=0,(H 2)$ and (H3) holds. According to Theorem 1, the problem (42) admits at least two nonzero weak solutions.

\section{Conclusions}

Fractional differential equations have recently proved to be valuable tools for modeling many phenomena in various fields of science and engineering. Indeed, one can find many applications in viscoelasticity, electrochemistry, control, porous media, electromagnetic, etc., for example, see [1-19]. Moreover, recently, the existence of solutions of boundary value problems for fractional differential equations have widely been studied in many papers and we refer the reader to the papers [3-18]. In this work, we can extend the previous mentioned works for proving the existence of at least two non zero weak solutions to a new class of impulsive fractional boundary value problems via Brezis and Nirenberg's Linking Theorem. Finally, an example is presented to illustrate our results. In the next work, we will try to prove the existence of three different weak solutions of the $p$-Laplacian fractional for an overdetermined nonlinear fractional partial Fredholm-Volterra integro-differential system by using variational methods combined with a critical point theorem due to Bonanno and Marano.

Author Contributions: Conceptualization, methodology and writing-original manuscript, A.A., R.G. and S.B.; supervision, S.B.; writing review and editing, S.B. and R.G., formal analysis, S.B. and A.A. All authors have read and agreed to the published version of the manuscript.

Funding: This research received NO external or internal funding.

Acknowledgments: The authors would like to thank the both anonymous referees for their careful reading and for relevant remarks/suggestions which helped them to improve the paper.

Conflicts of Interest: The authors declare no conflict of interest.

\section{References}

1. Agarwal, P.; Karimov, E.; Mamchuev, M.; Ruzhansky, M. On boundary-value problems for a partial differential equation with Caputo and Bessel operators. In Recent Applications of Harmonic Analysis to Function Spaces, Differential Equations, and Data Science; Birkhauser: Cham, Switzerland, 2017; Volume 152, pp. 707-718.

2. Atangana, A.; Hammouch, Z. Fractional calculus with power law: The cradle of our ancestors. Eur. Phys. J. Plus 2017, 134, 429. [CrossRef]

3. Bai, C. Existence of solutions for a nonlinear fractional boundary value problem via a local minimum theorem. Elect. J. Differ. Equat. 2012, 176, 1-9.

4. Benson, D.; Wheatcraft, S.; Meerschaert, M. Application of a fractional advection-dispersion equation. Water Resour. Res. 2000, 36, 1403-1412. [CrossRef]

5. Benson, D.; Wheatcraft, S.; Meerschaert, M. The fractional-order governing equation of Levy motion. Water Resour. Res. 2000, 36, 1413-1423. [CrossRef]

6. Boulaaras, S.; Bouizem, Y.; Guefaifia, R. Existence of positive solutions of $(p(x), q(x))$-Laplacian parabolic systems with right hand side defined as a multiplication of two separate functions. Math. Methods Appl. Sci. 2020, 43, 2615-2625. [CrossRef]

7. Boulaaras, S.; Allahem, A. Existence of Positive Solutions of Nonlocal $p(x)$-Kirchhoff Evolutionary Systems via Sub-Super Solutions Concept. Symmetry 2019, 11, 253. [CrossRef] 
8. Boulaaras, S.; Guefaifia, R. Existence of positive weak solutions for a class of Kirrchoff elliptic systems with multiple parameters. Math. Methods Appl. Sci. 2018, 41, 5203-5210. [CrossRef]

9. Diethelm, K.; Freed, A. On the solution of nonlinear fractional order differential equations used in the modeling of viscoelasticity. In Scientific Computing in Chemical Engineering II-Computational Fluid Dynamics, Reaction Engineering and Molecular Properties; Keil, F., Mackens, W., Voss, H., Werther, J., Eds.; Springer: Berlin/Heidelberg, Germany, 1999; pp. 217-224.

10. Devi, J.; Lakshmikantham, V. Nonsmooth analysis and fractional differential equations. Nonlinear Anal. TMA 2009, 70, 4151-4157. [CrossRef]

11. Erwin, V.; Roop, J. Variational formulation for the stationary fractional advection dispersion equation. Numer. Methods Partial Differ. Equ. 2006, 22, 58-76.

12. Fix, G.; Roop, J. Least squares finite-element solution of a fractional order two-point boundary value problem. Comput. Math. Appl. 2004, 48, 1017-1033. [CrossRef]

13. Glockle, W.; Nonnenmacher, T. A fractional calculus approach of self-similar protein dynamics. Biophys. J. 1995, 68, 46-53. . [CrossRef]

14. Guefaifia, R.; Boulaaras, S. Sub-super solutions method for elliptic systems involving $\left(p_{1}, \ldots, p_{m}\right)$ Laplacian operator. Math. Methods Appl. Sci. 2020, 43, 4191-4199.

15. Hilfer, R. Applications of Fractional Calculus in Physics; World Scientific: Singapore, 2000.

16. Jarad, F. Abdeljawad, T.; Hammouch, Z. On a class of ordinary differential equations in the frame of Atangana-Baleanu fractional derivative. Chaos Solitons Fractals 2018, 117, 16-20. [CrossRef]

17. Kamache, F.; Guefaifia, R.; Boulaaras, S.; Alharbi, A. Existence of weak solutions for a new class of fractional p-Laplacian boundary value systems. Mathematics 2020, 8, 475. [CrossRef]

18. Kirchner, J.; Feng, X.; Neal, C. Fractal streamchemistry and its implications for contaminant transport in catchments. Nature 2000, 403, 524-526. [CrossRef]

19. Kiymaz, I.O.; Cetinkaya, A.; Agarwal, P. An extension of Caputo fractional derivative operator and its applications. J.Nonlinear Sci. Appl. 2016, 9, 3611-3621. [CrossRef]

20. Torres, C.; Oliverio, D. Existence of three solution for fractional Hamiltonian system. J. Homepage 2017, 4, 51-58. [CrossRef]

21. Zhang, S. Positive solutions to singular boundary value problem for nonlinear fractional differential equation. Comput. Math. Appl. 2010, 59, 1300-1309. [CrossRef]

22. Zhou, J.; Wang, Y.; Li, Y. Existence of solutions for a class Of Fractional Hamiltonian Systems with impulsive effects. Fract. Differ. Calc. 2018, 8, 233-253. [CrossRef]

23. Brézis, H.; Nirenberg, L. Remarks on finding critical points. Commun. Pure Appl. Math, 1991, 44, 939-963. [CrossRef]

24. Podlubny, I. Fractional Differential Equations; Academic Press: San Diego, CA, USA, 1999.

25. Rodriguez-Lopez, R.; Tersian, S. Multiple solutions to boundary value problem for impulsive fractional differential equations. Fract. Calc. Appl. Anal. 2014, 17, 1016-1038. [CrossRef]

(C) 2020 by the authors. Licensee MDPI, Basel, Switzerland. This article is an open access article distributed under the terms and conditions of the Creative Commons Attribution (CC BY) license (http://creativecommons.org/licenses/by/4.0/). 